\title{
Utilization of a rapid response team and associated outcomes in patients with malignancy
}

\author{
Jongmin Lee', Woo Ho Ban², Sei Won Kim², Eun Young Kim³, Mi Ra Han", Seok Chan Kim¹ \\ ${ }^{1}$ Division of Pulmonary and Critical Care Medicine, Department of Internal Medicine, Seoul St. Mary's Hospital, College of Medicine, The Catholic University \\ of Korea, Seoul, ${ }^{2}$ Division of Pulmonary and Critical Care Medicine, Department of Internal Medicine, Eunpyeong St. Mary's Hospital, College of Medicine, \\ The Catholic University of Korea, Seoul; ${ }^{3}$ Division of Trauma and Surgical Critical Care, Department of Surgery, Seoul St. Mary's Hospital, College of Medicine, \\ The Catholic University of Korea, Seoul; ${ }^{4}$ St. Mary's Advanced Life Support Team, Department of Nursing, Seoul St. Mary's Hospital, College of Medicine, The \\ Catholic University of Korea, Seoul, Korea
}

Background: Recent advances in diagnosis and treatment have improved long-term outcomes in cancer patients. As a result, the requirement for a rapid response team (RRT) for cancer patients is also increasing. This study aimed to analyze utilization of an RRT and the associations between related factors and mortality in a population of cancer patients.

Methods: This retrospective cohort study included hospitalized patients at a single academic medical center in Seoul, Korea, who required RRT activation during a 6-year period from June 2013 to December 2018.

Results: Overall, 164 of the 457 patients who met the above criteria were cancer patients, and they had a significantly higher Charlson comorbidity score than the non-cancer patients (5.0 vs. 7.0, $P<0.001$ ). A significantly larger proportion of cancer patients required intensive care unit transfer ( $51.8 \%$ vs. $41.0 \%, \mathrm{P}=0.032$ ). Cancer patients also had significantly higher in-hospital mortality compared with other patients ( $39.6 \%$ vs. 10.9\%, $\mathrm{P}<0.001)$. Furthermore, presence of cancer was independently associated with in-hospital mortality (adjusted odds ratio [OR], 2.09; 95\% confidence interval [Cl], 1.11 to 3.93). Among cancer patients, higher Acute Physiology and Chronic Health Evaluation (APACHE) II at the time of RRT activation was significantly associated with in-hospital mortality regardless of malignancy (adjusted OR, $1.08 ; 95 \% \mathrm{Cl}, 1.01$ to 1.15$)$.

Conclusions: Cancer patients requiring RRT activation have significantly higher rates of inhospital mortality than patients not using RRT. Higher severity score at the time of RRT activation in patients with malignancy was significantly associated with in-hospital mortality.

Key Words: cancer; clinical deterioration; hospital rapid response team

\section{INTRODUCTION}

Rapid response teams (RRTs) are implemented in hospitals to prevent serious adverse events such as cardiac arrest, unexpected admission to the intensive care unit (ICU), and death [1]. Numerous single-center studies have reported associations of RRTs with reductions in inhospital mortality and cardiopulmonary arrest [2-5]. However, the potential impacts of RRT activation on in-hospital mortality and cardiopulmonary arrest remain controversial. Although a recent systematic review suggested associations of RRTs with reductions in in-hospital mor-

\section{Original Article}

Received: August 24, 2019

Revised: November 18, 2019

Accepted: February 19, 2020

Corresponding author

Seok Chan Kim

Division of Pulmonary and Critical

Care Medicine, Department of

Internal Medicine, Seoul St. Mary's

Hospital, College of Medicine, The

Catholic University of Korea, 222

Banpo-daero, Seocho-gu, Seoul

06591, Korea

Tel: +82-2-2258-6062

Fax: +82-2-2258-6062

E-mail: cmcksc@catholic.ac.kr

Copyright $(\bigodot 2020$ The Korean Society of Critical Care Medicine

This is an Open Access article distributed under the terms of Creative Attributions Non-Commercial License (https:// creativecommons.org/li-censes/by-nc/4.0/) which permits unrestricted noncommercial use, distribution, and reproduction in any medium, provided the original work is properly cited. 
tality and cardiopulmonary arrest [6], other meta-analyses of RRT studies reported only weak evidence to support an overall reduction in in-hospital mortality $[7,8]$. This discrepancy may be attributable to factors such as heterogeneity in study populations and differences in activation criteria, composition, and activation quality of RRTs.

Recent developments in early diagnosis and management of cancer have led to improvements in long-term prognosis of cancer patients [9]. Despite declining cancer death rates, however, malignancy-related complications or treatment-associated side effects remain clinically challenging and worsen patient prognoses. Previous studies reported an association between early intervention (i.e., before development of severe organ failure) and better outcomes despite the inherently high acuity level in this population $[10,11]$. Compared with noncancer patients, cancer patients experienced higher rates of in-hospital mortality after RRT activation [12]. Moreover, as RRTs are often activated during the end-of-life stage, RRT activation might play an important role in discussions regarding goals of care or initiation of palliative care for a cancer patient [12-14]. To date, few studies have investigated the utilization and outcomes of RRTs in cancer patients. Therefore, the current study aimed to analyze utilization of an RRT and the associations of related factors with mortality in a population of cancer patients.

\section{MATERIALS AND METHODS}

\section{Design}

We retrospectively reviewed the RRT activation records at Seoul St. Mary's Hospital from January 2014 to December 2018. Seoul St. Mary's Hospital is a tertiary teaching hospital in Korea that admits approximately 85,000 adults per year. Approval for this study was obtained from the Institutional Review Board of Seoul St. Mary's Hospital. The requirement for informed consent was waived by the ethical review board.

\section{Patients and Setting}

The study objective was to determine the utilization and outcomes of oncology patients who required RRT activation. The hospital RRT, which is called the St. Mary's Advanced Life Support Team, was implemented in June 2013 with the aims of reducing in-hospital mortality and improving the quality of hospital care. Since 2016, medical staff affiliated with the RRT have provided 24 hour/day coverage of all departments except pediatrics.

\section{KEY MESSAGES}

- In this study, oncology patients requiring rapid response team activation had significantly higher rates of in-hospital mortality.

- Moreover, among oncology patients, higher severity score at the time of rapid response team activation was significantly associated with in-hospital mortality regardless of malignancy.

\section{RRT Activation and Screening}

The criteria for RRT activation are shown in Table 1. In this study, we summarized and re-classified these criteria into seven categories: heart rate change, blood pressure change, respiratory rate change, $\mathrm{SaO}_{2}$ change, altered mental status, staff concern, and other reason. RRT nurses performed primary screening and checks and then called an RRT physician. RRT activation was defined as the process wherein intensivists or residents arrive at the patient's bedside and provide medical treatment, consultation, or decisions about transfer to the ICU. At each activation, the RRT transported a portable multi-monitor and the point-of-care blood analysis system (Epoc; Alere, Waltham, MA, USA). Patients were also protected by an electronic medical record (EMR)-based screening system and were automatically screened if a clinical value determined by the EMR satisfied RRT activation criteria. The RRT charge nurse assessed the condition of each patient and recommended RRT activation to the ward staff or nurses as needed.

Table 1. Criteria for RRT activation

\begin{tabular}{l} 
Criteria \\
\hline Respiratory rate $\leq 8 / \mathrm{min}$ or $\geq 25 / \mathrm{min}$ \\
$\mathrm{O}_{2}$ Saturation $\leq 90 \%$ for more than 5 minutes with prior oxygen therapy \\
$\mathrm{pH} \leq 7.3, \mathrm{PaCO}_{2} \geq 50 \mathrm{~mm} \mathrm{Hg}, \mathrm{PaO}_{2} \leq 55 \mathrm{~mm} \mathrm{Hg}$, lactic acid $\geq 2.0 \mathrm{mM} / \mathrm{L}$ \\
Stridor/accessory muscle use \\
Systolic blood pressure $\leq 85 \mathrm{~mm} \mathrm{Hg}$ with correlated symptoms or signs \\
Heart rate $\leq 50 /$ min or $\geq 130 / \mathrm{min}$ \\
Acute chest pain \\
Urine output $\leq 50 / \mathrm{ml}$ in 4 hours \\
Sudden alteration of consciousness \\
Unexplained agitation or anxiety \\
Seizure \\
Any serious concerns about overall deterioration \\
RRT: rapid response team; PaCO 2 partial pressure of carbon dioxide.
\end{tabular}




\section{Data Collection}

We collected data on baseline demographics, including age and sex, from the EMR database. Comorbidity data were collected for the studied patients and used to calculate the Charlson comorbidity score index [15]. For each patient, we also recorded hospital length of stay, number of days between admission and RRT activation, nature of the event leading to RRT activation, Acute Physiology and Chronic Health Evaluation (APACHE) II score, and Modified Early Warning Score (MEWS). The APACHE II scores were collected for all patients with RRT activation, regardless of ICU transfer. The study outcomes were survival and ICU transfer status within 24 hours after RRT activation.

\section{Statistical Analysis}

Statistically significant differences between groups were analyzed using the chi-square, Wilcoxon rank-sum, Student t-test, or Welch's test as appropriate. Factors associated with in-hospital mortality were assessed by logistic regression. Data are expressed as mean \pm standard deviation for normally distributed continuous variables and median and interquartile range for non-normally distributed continuous data. Categorical data are reported as number (\%). A two-tailed P-value $<0.05$ was considered statistically significant. All statistical analyses were performed using R 3.4.1 version (R Foundation, Vienna, Austria).

\section{RESULTS}

A total of 457 patients required RRT activation during the indicated study period, including 164 patients with cancer. Notably, utilization of RRT resources during the study period was

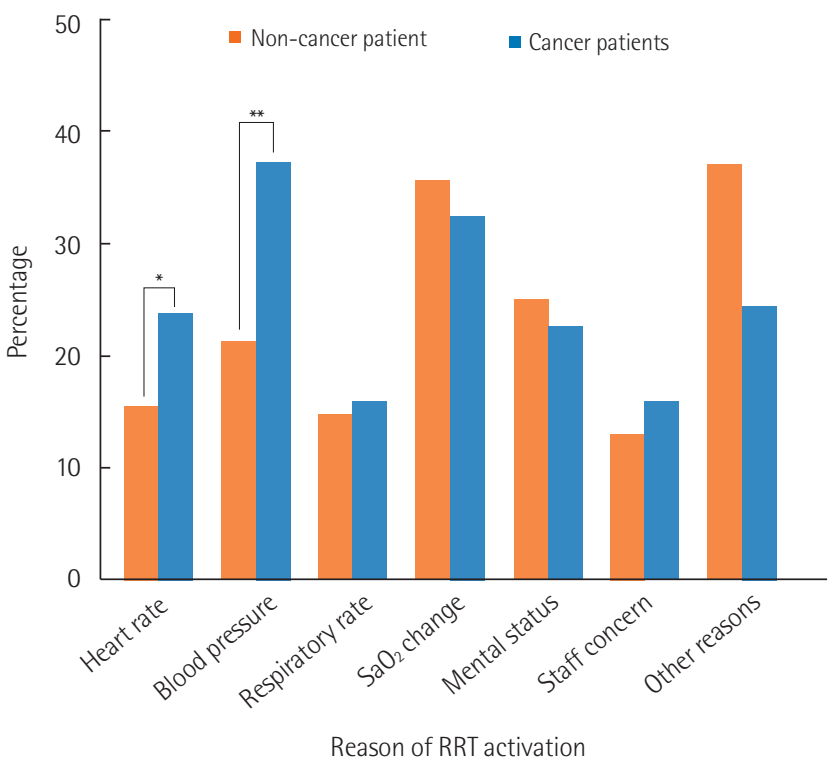

Figure 1. Reasons for rapid response team activation. Changes in heart rate and blood pressure were significantly more common in cancer patients than non-cancer patients. RRT: rapid response team. ${ }^{*} \mathrm{P}<0.05,{ }^{* *} \mathrm{P}<0.01$.

Table 2. Baseline patient characteristics

\begin{tabular}{lccr}
\hline Variable & Non-cancer patient $(n=293)$ & Cancer patient $(n=164)$ & P-value \\
\hline Male sex & $142(48.5)$ & $65(39.6)$ & 0.085 \\
Age $(y r)$ & $72.0(59.0-80.0)$ & $63.0(54.5-72.0)$ & $<0.001$ \\
Diabetes & $106(36.2)$ & $38(23.2)$ & 0.006 \\
Renal failure & $61(20.8)$ & $7(4.3)$ & $<0.001$ \\
Congestive heart failure & $57(19.5)$ & $8(4.9)$ & $<0.001$ \\
History of myocardial infarction & $60(20.5)$ & $10(6.1)$ & $<(8.5)$ \\
Cerebrovascular disease & $51(17.4)$ & $9(5.5)$ & 0.001 \\
Peripheral vascular disease & $33(11.3)$ & $11(6.7)$ & 0.014 \\
Moderate to severe liver disease & $10(3.4)$ & $5(3.0)$ & 0.060 \\
Dementia & $35(11.9)$ & $8(4.9)$ & 0.167 \\
Connective tissue disease & $20(6.8)$ & $2(1.2)$ & 0.002 \\
Hemiplegia & $13(4.4)$ & $7.0(6.0-9.0)$ & 0.529 \\
Charlson comorbidity score & $5.0(3.0-6.0)$ & $16.0(10.0-20.0)$ & 0.117 \\
APACHE II score & $14.0(9.0-17.0)$ & $7.0(5.0-8.0)$ & $<0.001$ \\
MEWS & $5.0(4.0-7.0)$ & 0.007 & $<0.001$ \\
\hline
\end{tabular}

Values are presented as number (\%) or median (interquartile range).

APACHE: Acute Physiology and Chronic Health Evaluation; MEWS: Modified Early Warning Score. 
higher for cancer patients than non-cancer patients, with 2.80 vs. 1.38 per 1,000 patient discharges. Table 2 presents the baseline characteristics of the included patients. Non-cancer patients were younger and less likely to have diabetes, renal failure, congestive heart failure, history of myocardial infarction, cerebrovascular disease, and dementia compared to cancer patients. Cancer patients had a significantly higher Charlson comorbidity score $(\mathrm{P}<0.001$ vs. non-cancer patients $)$ and higher severity scores of APACHE II and MEWS ( $\mathrm{P}=0.007$ and $\mathrm{P}<0.001$, respectively).

Figure 1 depicts the reasons for RRT activation. A change in blood pressure was the most common reason for RRT activation among cancer patients, whereas a change in $\mathrm{SaO}_{2}$ was the most common reason among non-cancer patients. Changes in heart rate and blood pressure were significantly more com- monly recorded for cancer patients vs. non-cancer patients (23.8\% vs. $15.4 \%, \mathrm{P}=0.037$ and $37.2 \%$ vs. $21.2 \%, \mathrm{P}<0.001$, respectively).

Table 3 lists the patient outcomes after RRT activation. In this study, cancer patients had a significantly higher rate of inhospital mortality compared with non-cancer patients (39.6\% vs. $10.9 \%, \mathrm{P}<0.001)$. Moreover, a significantly larger proportion of cancer patients required ICU transfer (51.8\% vs. $41.0 \%$, $\mathrm{P}=0.032)$. Only two cancer patients $(1.2 \%)$ had a preexisting do-not-resuscitate (DNR) order. Within 24 hours after RRT activation, 24 patients (14.6\%) experienced a change in code status, and palliative care involvement after RRT activation was reported for only 11 patients (10.6\%).

The presence of cancer was independently associated with in-hospital mortality on multivariate analysis (adjusted odds

Table 3. Outcome after rapid response team activation

\begin{tabular}{lccr}
\hline Variable & Non-cancer patient $(n=293)$ & Cancer patient $(n=164)$ & P-value \\
\hline In-hospital mortality & $32(10.9)$ & $65(39.6)$ & $<0.001$ \\
ICU transfer required & $120(41.0)$ & $85(51.8)$ & 0.032 \\
In-hospital mortality if ICU transfer required & $21(17.5)$ & $39(45.9)$ & $<0.001$ \\
Pre-existing do-not-resuscitate & 0 & $2(1.2)$ & 0.248 \\
Code status change within 24 hours after RRT & $4(1.4)$ & $11(10.6)$ & $<0.001$ \\
Palliative care involvement after RRT & $1(0.6)$ & $<0.001$ \\
\hline
\end{tabular}

Values are presented as number (\%).

ICU: intensive care unit; RRT: rapid response team.

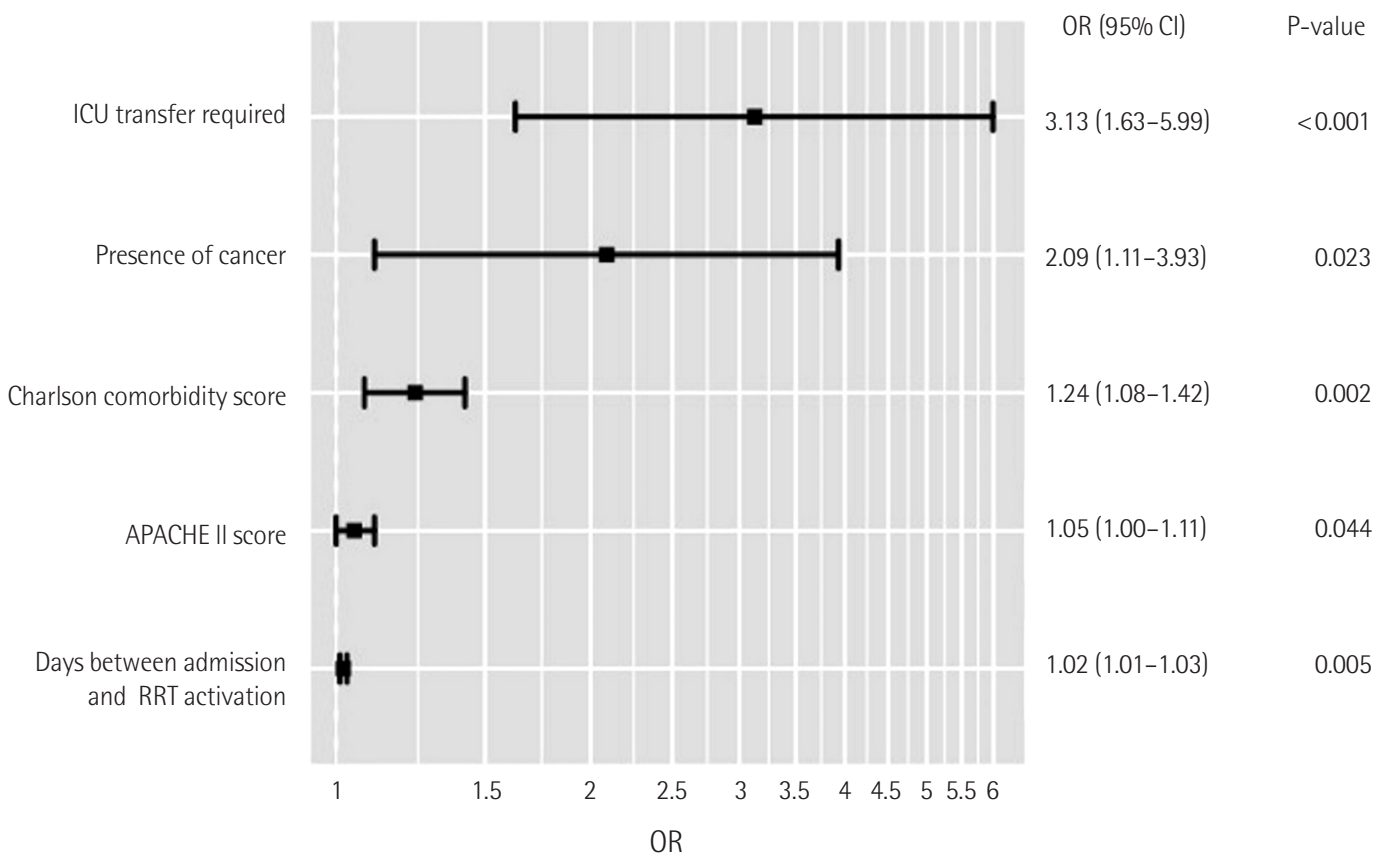

Figure 2. Multivariate analysis for factors independently associated with in-hospital mortality. ICU: intensive care unit; APACHE: Acute Physiology and Chronic Health Evaluation; RRT: rapid response team; OR: odds ratio; Cl: confidence interval. 
Table 4. Factors associated with survival in cancer patients

\begin{tabular}{lccc}
\hline Variable & Survivor $(n=97)$ & Non-survivor $(n=41)$ & P-value \\
\hline Male sex & $42(43.3)$ & $14(34.1)$ & 0.417 \\
Age $(\mathrm{yr})$ & $63.0(55.0-72.0)$ & $18(51.0-72.0)$ & 0.843 \\
Chemotherapy in last 30 days & $35(36.1)$ & $8.0(6.0-9.0)$ & 0.502 \\
Charlson comorbidity score & $7.0(6.0-8.0)$ & $17.0(13.0-22.0)$ & 0.067 \\
APACHE II score & $14.0(9.0-18.5)$ & $7.0(5.5-9.0)$ & 0.018 \\
MEWS & $6.0(4.0-8.0)$ & $9.0(3.0-26.0)$ & 0.004 \\
Day between admission and RRT activation & $4.0(2.0-13.0)$ & $29(70.7)$ & 0.032 \\
ICU transfer & $46(47.4)$ & $10(24.4)$ & 0.020 \\
Status of malignancy & & $21(51.2)$ & 0.742 \\
First presentation & $28(28.9)$ & $21(51.2)$ & 0.657 \\
Relapse/refractory & $44(45.4)$ & $9.0(2.0-32.0)$ & 0.263 \\
Extensive disease & $38(39.2)$ & & \\
Duration of malignancy & $13.0(1.0-48.0)$ & & \\
\hline
\end{tabular}

Values are presented as number (\%) or median (interquartile range).

APACHE: Acute Physiology and Chronic Health Evaluation; MEWS: Modified Early Warning Score; RRT: rapid response team; ICU: intensive care unit.

aPatients who had a preexisting do-not-resuscitate order or change in code status within 24 hours post RRT activation were excluded from analysis.

Table 5. Multivariable analyses with logistic regression models for probability of in-hospital mortality

\begin{tabular}{lcc}
\hline Variable & $\begin{array}{c}\text { Adjusted odds ratio } \\
(95 \% \mathrm{Cl})\end{array}$ & P-value \\
\hline Age (yr) & $0.98(0.94-1.02)$ & 0.405 \\
Chemotherapy in last 30 days & $0.69(0.24-1.97)$ & 0.492 \\
Charlson comorbidity score & $1.20(0.92-1.55)$ & 0.175 \\
APACHE II score & $1.08(1.01-1.15)$ & 0.027 \\
MEWS & $1.02(0.82-1.27)$ & 0.838 \\
Days between admission and RRT & $1.01(1.00-1.03)$ & 0.166 \\
$\quad$ activation & $2.52(1.04-6.11)$ & 0.040 \\
ICU transfer required & & \\
Status of malignancy & $0.76(0.24-2.47)$ & 0.653 \\
Relapse/refractory & $1.92(0.79-4.66)$ & 0.151 \\
Extensive disease & $0.99(0.97-1.00)$ & 0.135 \\
\hline Duration of malignancy &
\end{tabular}

Patients who had a preexisting do-not-resuscitate order or a change in code status within 24 hours post RRT activation were excluded from analysis.

Cl: confidence interval; APACHE: Acute Physiology and Chronic Health Evaluation; MEWS: Modified Early Warning Score; RRT: rapid response team; ICU: intensive care unit.

ratio [OR], 2.09; 95\% confidence interval [CI], 1.11 to 3.93; $\mathrm{P}=0.023$ ). Patients with a preexisting DNR order or who experienced a change in code status within 24 hours after RRT activation were excluded from the analysis. Other independent risk factors for hospital mortality were ICU transfer (adjusted
OR, 3.13; 95\% CI, 1.63 to 5.99; $\mathrm{P}<0.001$ ), Charlson comorbidity score (adjusted OR, 1.24; 95\% CI, 1.08 to 1.42; $\mathrm{P}<0.001$ ), APACHE-II score (adjusted OR, 1.05; 95\% CI, 1.00 to $1.11 ; \mathrm{P}=0.0437$ ), and number of days between admission and RRT activation (adjusted OR, 1.02; 95\% CI, 1.01 to 1.03; $\mathrm{P}=0.005$ ) (Figure 2).

Table 4 lists the demographic differences between survivors and non-survivors among patients with malignancy. Patients with a preexisting DNR order and those who experienced a change in code status within 24 hours after RRT activation were excluded from this analysis. Notably, in cancer patients, non-survivors had significantly higher APACHE-II score and MEWS and a longer interval between admission and RRT activation than survivors. Unsurprisingly, a significantly larger proportion of patients requiring ICU transfer was observed among non-survivors relative to survivors. After adjusting for potential confounding factors, APACHE II score remained significantly associated with in-hospital mortality (adjusted OR, 1.08; 95\% CI, 1.01 to 1.15; $\mathrm{P}=0.027$ ). ICU transfer was also independently associated with in-hospital mortality (adjusted OR, 2.52; 95\% CI, 1.04 to 6.11, $\mathrm{P}=0.040$ ) (Table 5).

\section{DISCUSSION}

In this study, which aimed to evaluate the utilization and outcomes of RRT and the factors associated with mortality in cancer patients, we found that cancer patients had significantly higher rates of in-hospital mortality and ICU transfer than 
non-cancer patients. Furthermore, ICU transfer was associated with a higher mortality rate among cancer patients.

Although RRTs have been widely adopted by health systems over the last 20 years [16], previous studies have failed to clarify the effectiveness of these teams for reducing hospital mortality [6], possibly due to discrepancies in study populations, criteria for RRT activation, and quality of RRT activation. Moreover, major cancer centers have implemented RRTs along with advances in chemotherapy and trends in provision of increasingly aggressive treatments $[13,17]$. However, a previous study reported that adverse outcomes are more common among cancer patients requiring RRT than among non-cancer patients [17].

In this study, cancer patients were more likely to require RRT activation during the study period and ICU transfer after an RRT event compared to their counterparts without cancer. Additionally, cancer patients also had significantly higher disease severity scores (APACHE II score, MEWS) and Charlson comorbidity score. As expected, cancer patients requiring RRT had a significantly higher rate of in-hospital mortality compared to their counterparts without cancer, and this association remained significant after adjusting for confounding factors, including severity scores and Charlson comorbidity score. These findings suggest inherently higher mortality rates among cancer patients, especially those experiencing acute medical deterioration, than among non-cancer patients. These results are consistent with the findings of previous studies in which cancer patients were found to have an inherently high acuity level [15,18].

In this study, the in-hospital mortality rate was significantly higher among cancer patients who required ICU transfer after an RRT event than among non-cancer patients, consistent with previous studies $[12,19,20]$. Therefore, determination of key prognostic predictors of poor outcome is crucial to identifying patients who may benefit from ICU admission. In the present study, higher severity scores at the time of RRT activation, especially the APACHE II score, were significantly associated with in-hospital mortality after adjusting for potential confounding factors in cancer patients. The APACHE II scoring system is an accurate measurement of clinical severity and correlates strongly with outcome in critically ill patients [21]. Consistent with our results, previous studies demonstrated effectiveness of APACHE II score for predicting in-hospital mortality of critically ill patients with cancer in need of intensive care [22,23]. Our findings emphasize that the prognosis of cancer patients is determined by severity at the time of RRT activation regardless of malignancy status.
Recently, several studies have emphasized the role of RRT in end-of-life care provided to cancer patients. Although the RRT primarily aims to detect reversible deterioration, the death process is a frequent trigger of RRT activation [13]. One previous large, multi-center, randomized controlled trial observed an association of RRTs with increase in DNR orders [24], while Coventry et al. [25] reported that RRT calls for patients with pre-existing DNR orders are not uncommon. In the latter study, $15.7 \%$ of patients with a pre-established DNR order required RRT activation. Furthermore, a recent review indicated that up to one-third of RRT calls involved patients receiving endof-life care [13]. Consistent with those previous studies, we found that a fairly large number of RRT events involved patients receiving end-of-life care. Specifically, $14.6 \%$ of cancer patients showed a change in code status within 24 hours after RRT, and $10.6 \%$ were receiving palliative care. Therefore, a better understanding of the role of the RRT during provision of end-of-life care is needed to ensure that patients receive appropriate palliative and comfort care.

The present study had several limitations. First, this was a retrospective study with a relatively small number of patients. Therefore, selection bias may have affected the significance of our findings. Furthermore, our study was conducted at a single institution, which may have limited the generalizability of our findings. Second, this study did not evaluate the time to intervention, defined as the interval from the earliest time when the screening criteria were met until the time when RRT management was initiated $[11,26]$. However, previous studies reported that early recognition and timely treatment of patients were important contributors to the success of RRT $[1,11]$. Further studies are warranted to determine the impact of time to intervention in cancer patients who require RRT.

In conclusion, cancer patients in this study who required RRT had significantly higher rates of mortality and ICU transfer than non-cancer patients. The presence of cancer was independently associated with mortality. In patients with malignancy, higher severity scores were an independent predictor of in-hospital mortality. Considering the increasing utilization and expanding role of RRT in cancer patients, further research should focus on identifying subgroups of patients who would most benefit from ICU admission or palliative care services.

\section{CONFLICT OF INTEREST}

No potential conflict of interest relevant to this article was reported. 


\section{ORCID}

Jongmin Lee

Woo Ho Ban

Sei Won Kim

Eun Young Kim

Mi Ra Han

Seok Chan Kim https://orcid.org/0000-0002-0165-5856

https://orcid.org/0000-0002-7115-0490

https://orcid.org/0000-0002-2798-421X

https://orcid.org/0000-0003-4408-4165

https://orcid.org/0000-0001-9501-2087

https://orcid.org/0000-0002-0054-5962

\section{AUTHOR CONTRIBUTIONS}

Conceptualization: SCK. Data curation: JL, SWK. Formal analysis: WHB. Methodology: EYK. Project administration: JL. Visualization: MRH. Writing - original draft: JL. Writing - review \& editing: WHB, SCK.

\section{REFERENCES}

1. Devita MA, Bellomo R, Hillman K, Kellum J, Rotondi A, Teres $D$, et al. Findings of the first consensus conference on medical emergency teams. Crit Care Med 2006;34:2463-78.

2. Bellomo R, Goldsmith D, Uchino S, Buckmaster J, Hart GK, Opdam $\mathrm{H}$, et al. A prospective before-and-after trial of a medical emergency team. Med J Aust 2003;179:283-7.

3. Chen J, Bellomo R, Flabouris A, Hillman K, Finfer S; MERIT Study Investigators for the Simpson Centre, et al. The relationship between early emergency team calls and serious adverse events. Crit Care Med 2009;37:148-53.

4. Buist MD, Moore GE, Bernard SA, Waxman BP, Anderson JN, Nguyen TV. Effects of a medical emergency team on reduction of incidence of and mortality from unexpected cardiac arrests in hospital: preliminary study. BMJ 2002;324:387-90.

5. Jones D, Bellomo R, Bates S, Warrillow S, Goldsmith D, Hart $\mathrm{G}$, et al. Long term effect of a medical emergency team on cardiac arrests in a teaching hospital. Crit Care 2005;9:R80815.

6. Maharaj R, Raffaele I, Wendon J. Rapid response systems: a systematic review and meta-analysis. Crit Care 2015;19:254.

7. Chan PS, Jain R, Nallmothu BK, Berg RA, Sasson C. Rapid response teams: a systematic review and meta-analysis. Arch Intern Med 2010;170:18-26.

8. Winters BD, Pham JC, Hunt EA, Guallar E, Berenholtz S, Pronovost PJ. Rapid response systems: a systematic review. Crit Care Med 2007;35:1238-43.

9. Brenner H. Long-term survival rates of cancer patients achieved by the end of the 20th century: a period analysis. Lancet 2002; 360:1131-5.
10. Azoulay E, Soares M, Darmon M, Benoit D, Pastores S, Afessa B. Intensive care of the cancer patient: recent achievements and remaining challenges. Ann Intensive Care 2011;1:5.

11. Song JU, Suh GY, Park HY, Lim SY, Han SG, Kang YR, et al. Early intervention on the outcomes in critically ill cancer patients admitted to intensive care units. Intensive Care Med 2012;38:1505-13.

12. Austin CA, Hanzaker C, Stafford R, Mayer C, Culp L, Lin FC, et al. Utilization of rapid response resources and outcomes in a comprehensive cancer center. Crit Care Med 2014;42:905-9.

13. Jones D, Moran J, Winters B, Welch J. The rapid response system and end-of-life care. Curr Opin Crit Care 2013;19:616-23.

14. Dargin JM, Mackey CG, Lei Y, Liesching TN. Resource utilization and end-of-life care in a US hospital following medical emergency team-implemented do not resuscitate orders. J Hosp Med 2014;9:372-8.

15. Charlson ME, Pompei P, Ales KL, MacKenzie CR. A new method of classifying prognostic comorbidity in longitudinal studies: development and validation. J Chronic Dis 1987;40:37383.

16. Lee A, Bishop G, Hillman KM, Daffurn K. The medical emergency team. Anaesth Intensive Care 1995;23:183-6.

17. Jones D, Warrillow S. Clinical deterioration in cancer patients: the role of the rapid response team. Crit Care Med 2014;42: 997-8.

18. Needham DM, Scales DC, Laupacis A, Pronovost PJ. A systematic review of the Charlson comorbidity index using Canadian administrative databases: a perspective on risk adjustment in critical care research. J Crit Care 2005;20:12-9.

19. Staudinger T, Stoiser B, Müllner M, Locker GJ, Laczika K, Knapp $\mathrm{S}$, et al. Outcome and prognostic factors in critically ill cancer patients admitted to the intensive care unit. Crit Care Med 2000;28:1322-8.

20. Taccone FS, Artigas AA, Sprung CL, Moreno R, Sakr Y, Vincent JL. Characteristics and outcomes of cancer patients in European ICUs. Crit Care 2009;13:R15.

21. Knaus WA, Draper EA, Wagner DP, Zimmerman JE. APACHE II: a severity of disease classification system. Crit Care Med 1985;13:818-29.

22. Xing X, Gao Y, Wang H, Huang C, Qu S, Zhang H, et al. Performance of three prognostic models in patients with cancer in need of intensive care in a medical center in China. PLoS One 2015;10:e0131329.

23. Abbott RR, Setter M, Chan S, Choi K. APACHE II: prediction of outcome of 451 ICU oncology admissions in a community hospital. Ann Oncol 1991;2:571-4.

24. Hillman K, Chen J, Cretikos M, Bellomo R, Brown D, Doig G, 
et al. Introduction of the medical emergency team (MET) system: a cluster-randomised controlled trial. Lancet 2005;365: 2091-7.

25. Coventry C, Flabouris A, Sundararajan K, Cramey T. Rapid response team calls to patients with a pre-existing not for re- suscitation order. Resuscitation 2013;84:1035-9.

26. Sebat F, Musthafa AA, Johnson D, Kramer AA, Shoffner D, Eliason $\mathrm{M}$, et al. Effect of a rapid response system for patients in shock on time to treatment and mortality during 5 years. Crit Care Med 2007;35:2568-75. 